\title{
Supercover 3D Polygon
}

\author{
Eric ANDRES ${ }^{+*}$, Claudio SIBATA ${ }^{+}$, Raj ACHARYA* \\ + Roswell Park Cancer Institute \\ Department of Radiation Medecine \\ Elm \& Carlton sts. Buffalo. NY 14263. USA \\ * University of New-York at Buffalo \\ Department of ECE. BioMedical Imaging Group. \\ Bell Hall 201. Buffalo. NY 14260. USA \\ e-mail: andres@rtplan.med.buffalo.edu
}

\begin{abstract}
A new discrete 3D polygon called Supercover Polygon is introduced. The polygon is a tunnel free plane segment defined by vertices and edges. An edge is a $3 \mathrm{D}$ line segment. Two different polygons can share a common edge and if they do, the union of both polygons is tunnel free. This is definition of discrete polygons that has the "most" properties in common with the continuous polygon. It seems particularly interesting for modelization of discrete scenes by way of digitization.
\end{abstract}

\section{Introduction}

The need for a discrete modeling environment emerges in many different fields : in medical imaging with reconstruction problems, in flight simulation for scene modeling, in the gaming industry, etc. One key element for that is the discrete 3D polygon. In this paper a new 3D discrete polygon definition, the Supercover Polygon, is introduced. The supercover polygon seems to be an interesting candidate for the digitization of continuous polygons because of it's many properties in common with it. The Supercover polygon has the following properties:

- the polygon is a $3 \mathrm{D}$ plane segment;

- the polygon is tunnel free;

- the polygon is defined by a sequence of vertices all belonging to the same continuous plane;

- two consecutive vertices are linked by an edge which is a discrete 3D line segment. They separate the interior of the discrete polygon from the rest of the discrete plane;

- if two polygons share a common edge then the union of the two polygons is tunnel free.

The Supercover has many properties of the continuous Euclidean 3D polygon. This is not the case with classical 3D polygons [4]. Classically 3D polygons are defined as 2-dimensional separating objects (no 6-connected holes) and not as tunnel free objects (without any holes at all). The problem is that it is difficult to connect two classical polygons and to conserve the 2-dimensional (6-connected) 
separation. Another problem is that in many applications 2-dimensional separation is not enough since there are still 1-dimensional (18-connected) and 0-dimensional (26-connected) tunnels. In discrete ray tracing for instance a 0 dimensional ray can pass a wall formed by these classical polygons. This is a serious problem for such applications. These and other problems are avoided by the Supercover Polygon.

In section 2 we present the notations and principal definitions used in this paper. In section 3 we deal with the definition and properties of objects defined as supercover of a continuous object. The Supercover polygon is introduced. We conclude in section 4 .

\section{Preliminaries}

$[\cdot]$ is the Integer Part Function Invariant by Translation (i.e. $[x]$ is the greatest integer smaller or equal to $x[2]) .\{x\}$, where $x$ is a real or rational value, is the Euclidean remainder $\{x\}=x-[x]$. The greatest common divisor for three integers $a, b, c$ is noted $G C D(a, b, c) . A \biguplus B$ stands for $A \cup B$ where $A \cap B=\emptyset$. $\mathbb{Z}$ is the set of the integers, $\mathbb{N}$ the set of the positive integers, $\mathbb{N}^{*}$ the set of the strictly positive integers and $\mathbb{R}$ the set of the real numbers. A discrete point is a point in $\mathbb{Z}^{3}$. A digital point is a point in $\mathbb{R}^{3}$ with integer coordinates. A discrete (resp. digital) object is a set of discrete (resp. digital) points. A voxel $V$ is a unit cube $\left[x-\frac{1}{2}, x+\frac{1}{2}\right] \times\left[y-\frac{1}{2}, y+\frac{1}{2}\right] \times\left[z-\frac{1}{2}, z+\frac{1}{2}\right]$ centered on a digital point of coordinates $(x, y, z)$. Each voxel has a unique corresponding discrete point and vice-versa.

The notation used in this paper to describe the connectivity relations is different from the classical 3D one. The proposed notation is more consistent with extensions to higher dimensions [1]. Two grid points $A\left(a_{1}, a_{2}, a_{3}\right)$ and $B\left(b_{1}, b_{2}, b_{3}\right)$ are said to be $N$-dimensional neighbors $\left(N=0,1\right.$ or 2 ) if $e_{i}=\left|a_{i}-b_{i}\right| \leq 1$ and if $N \leq 3-e_{1}-e_{2}-e_{3}$. The definition of $N$-dimensional connectivity, $N$-dimensional arcs and $N$-dimensional paths are immediately deduced from this notation. A discrete object has a $k$-dimensional tunnel if a $k$-dimensional arc can go "through" the discrete object without intersection. A more formal definition can be found in [3]. A discrete object is said to be $k$-dimensional separating if the object has no k-dimensional tunnel. A discrete object that is 0-dimensional separating is simply said to be tunnel free. The supercover $S^{w}$ of a continuous object $S$ are all the discrete points with corresponding voxel $V$ such that $V \cap S \neq \emptyset[3]$. The supercover has some interesting properties such as : $(S \cup T)^{w}=S^{w} \cup T^{w}$ and $S \subset T \Rightarrow S^{w} \subset T^{w}$.

A discrete plane is defined analytically as the discrete points solutions of a double Diophantine inequality. The following definition has been introduced by J-P. Réveillès [5].

Definition 1. Discrete plane in dimension 3

A discrete plane in dimension 3 is defined by: 


$$
\begin{gathered}
P(d, a, b, c, \omega)=\left\{(x, y, z) \in \mathbb{Z}^{3} \mid a x+b y+c z+d \epsilon[0, \omega[\}\right. \\
=\left\{(x, y, z) \in \mathbb{Z}^{3} \mid\left[\frac{a x+b y+c z+d}{\omega}\right]=0\right\}
\end{gathered}
$$

where $a, b, c, d \in \mathbb{Z} ; \omega \in \mathbb{N}^{*}$ and $\operatorname{GCD}(a, b, c)=1$.

$a, b, c$ are the coefficients of the discrete plane. $\omega$ is called the arithmetical thickness and $d$ the translation constant of the plane. The value $a x+b y+c z+d$ is called the control value of the discrete plane. A plane segment is a connected subset of a plane.

We can assume without loss of generality that $G C D(a, b, c)=1$ because if $G C D(a, b, c)=u>1$, then $P(d, a, b, c, \omega)=P\left(\left[\frac{d}{u}\right], \frac{a}{u}, \frac{b}{u}, \frac{c}{u}, \omega^{\prime}\right)$, where $\omega^{\prime}=$ $\left[\frac{\omega}{u}\right]+1$ if $\left\{\frac{\omega}{u}\right\}>\left\{\frac{d}{u}\right\}$ and $\omega^{\prime}=\left[\frac{\omega}{u}\right]$ else.

We can also assume without loss of generality that the coefficients defining a discrete plane are integers since we are only interested here in plane segments (polygons) of finite size. Let us suppose that we have a discrete plane $P=P(d, a, b, c, \omega)$ defined with coefficients $a, b, c, d \in \mathbb{R}^{3} ; \omega \in \mathbb{N}^{*}$ and that we are only considering a finite part of space $I=[0, p]^{3}$. The coefficients are decomposed as follow $a=a_{0}+\frac{a_{1}}{4 \rho}+a_{2}, b=b_{0}+\frac{b_{1}}{4 \rho}+b_{2}, c=c_{0}+\frac{c_{1}}{4 \rho}+c_{2}, d=d_{0}+\frac{d_{1}}{4 \rho}+d_{2}$ where $a_{0}, a_{1}, b_{0}, b_{1}, c_{0}, c_{1}, d_{0}, d_{1}, \rho$ are integers and $a_{2}, b_{2}, c_{2}, d_{2}$ positive real then it is easy to see that $P=P\left(4 \rho d_{0}+d_{1}, 4 \rho a_{0}+a_{1}, 4 \rho b_{0}+b_{1}, 4 \rho c_{0}+c_{1}, 4 \rho \omega\right)$ on $I$.

\section{Supercover Polygon}

Proposition 2. Supercover of a continuous plane

Let $u s$ consider the continuous $3 D$ plane $P$ defined by $a x+b y+c z+d=0$, with $a, b, c, d \in \mathbb{Z}$.

The supercover $P^{w}$ of the continuous plane $P$ is defined by the discrete points:

$$
\begin{aligned}
& \text { - If }|a|+|b|+|c| \text { is odd then } P^{w}=P\left(d+\left[\frac{|a|+|b|+|c|}{2}\right], a, b, c,|a|+|b|+|c|\right) \\
& \text { - else } P^{w}=P\left(d+\frac{|a|+|b|+|c|}{2}, a, b, c,|a|+|b|+|c|+1\right) .
\end{aligned}
$$

This property makes the link between the Supercover and the analytical defined discrete plane. The optimal arithmetical thickness for a tunnel free discrete plane is $|a|+|b|+|c|[1]$ : a discrete plane if not tunnel free for an arithmetical thickness smaller than $|a|+|b|+|c|$ and if one arbitrary point is removed from the plane a tunnel is created. This means that the Supercover is tunnel free and that for $|a|+|b|+|c|$ even, the supercover plane is not of optimal thickness. If a point verifying $a x+b y+c z= \pm \frac{|a|+|b|+|c|}{2}$ is removed the remaining discrete object is still tunnel free. 
Proof. : For what follows, we suppose without loss of generality that $0 \leq a \leq$ $b \leq c$. Let us call $P^{\prime}$ the discrete planes presented in the proposition as being equal to $P^{w}$.

Let us first consider the case where $a+b+c=2 e+1$ is odd. The arithmetical thickness of $P^{\prime}$ is $\omega=2 e+1$.

Is $P^{w} \subset P^{\prime}$ ? The closest integer points to the continuous plane who do not belong to $P^{\prime}$ are the points solution of $a x+b y+c z+d= \pm(e+1)$. Let's consider a point $A(t, u, v)$ solution for $e+1$. It is easy to see that the closest point of the voxel corresponding to $A$ to the continuous plane is $B\left(t-\frac{1}{2}, u-\frac{1}{2}, v-\frac{1}{2}\right)$, and we have $a\left(t-\frac{1}{2}\right)+\left(u-\frac{1}{2}\right)+\left(v-\frac{1}{2}\right)+d=e+1-e=1$. This means that $P$ does not cut the voxel corresponding to $A$. The same is true for $-(e+1)$. This proves that $P^{n w} \subset P^{\prime}$.

Is $P^{\prime} \subset P^{w}$ ? Let's consider a point $A(t, u, v) \in P^{\prime}$.

We have $B\left(t-\frac{1}{2}, u-\frac{1}{2}, v-\frac{1}{2}\right)$, one vertex of the voxel corresponding to $A$, such that $a\left(t-\frac{1}{2}\right)+\left(u-\frac{1}{2}\right)+\left(v-\frac{1}{2}\right)+d=a t+b u+c v+d-e \leq 0$, and $C\left(t+\frac{1}{2}, u+\frac{1}{2}, v+\frac{1}{2}\right)$, a second vertex, such that $a\left(t+\frac{1}{2}\right)+\left(u+\frac{1}{2}\right)+\left(v+\frac{1}{2}\right)+$ $d=a t+b u+c v+d+e \geq 0$. This means that the voxel corresponding to $A$ is cut by $P$. This achieves the proof for the case where $\omega$ is odd that $P^{\prime}=P^{w}$. Let us now consider the case where $a+b+c=2 e$ is even. We have then $\omega=2 e+1$ which leads to the same demonstration as above.

Let us now define a $3 \mathrm{D}$ discrete line segment :

Definition 3. Discrete 3D line segment

The 3D discrete line segment between the points $A$ and $B$ is the supercover $A B^{w}$ of the continuous line segment $A B$.

Proposition 4. link between discrete line segment and plane. The intersection of the supercovers of all the Euclidean planes containing $A B$ is $A B^{w}$.

This property is the basis of the Supercover Polygon definition. It means that whatever orientation the discrete polygon has, the edge does not change, therefore two different polygons will be able to share a common edge.

Proof. : Cohen-Kaufman have proved that a supercover is unique [3] which proves that $A B^{w}$ is included in the intersection of the supercovers of the planes. Let's now imagine a discrete point $C$ that belongs to the intersection of the supercovers and that does not belong to $A B^{w}$. Let's call $L$ the closest point of the voxel corresponding to $C$ to the segment $A B$ and $M$ the closest point of $A B$ to the voxel. We can define a continuous plane containing $A B$ and orthogonal to $L M$. This plane does not cut the voxel corresponding to $C$ and so $C$ can't belong to the intersection of all the supercovers. 
Proposition 5. Line inclusion

Let's consider the continuous segment $A B$ and a point $C$ of the segment, then the discrete $3 D$ line $A C^{w}$ and $C B^{w}$ are included in the discrete line $A B^{w}$, and $A B^{w}=A C^{w} \cup C B^{w}$.

The proof is obvious. The $2 \mathrm{D}$ version of this property is also interesting to consider for other applications.

Definition 6. Discrete 3D polygon

Let's consider a sequence of $n \geq 3$ points $\left(A_{0}, \ldots, A_{k}\left(x_{k}, y_{k}, z_{k}\right), \ldots, A_{n-1}\right)$ such that $a x_{k}+b y_{k}+c z_{k}+d=0$.

The Supercover Polygon is defined as the supercover of the continuous polygon defined by the sequence of points. We call edge of the discrete polygon the discrete line segment $A_{i} A_{i+1}$ (with $A_{n}=A_{0}$ ) and we call interior of the discrete polygon all the points of the polygons who do not belong to an edge.

Note that the interior of a discrete $3 \mathrm{D}$ polygon can be empty.

Proposition 7. Properties of the discrete $3 D$ polygon

The discrete $3 D$ polygon is a discrete plane segment, is tunnel free and the union of two $3 D$ discrete polygons sharing a common edge $A B$ is tunnel free.

Proof of Proposition 7 is simple. We can use proposition 5 to connect without problems 3D polygons who are not sharing a complete common edge. This can be very useful for the modelization of complex discrete scenes.

Proposition 8. Separation property

Let's consider a discrete polygon based on a discrete plane $P$. The edges of the polygon 0-dimensional separate the interior of the polygon from the rest of $P$.

0-dimensional separation means here that no 0-dimensional paths within $P$ can cross the border (here the edge) without intersection. Cohen-Kaufman proved that a supercover is 0-dimensional separating [3]. This is a direct application of their result.

\section{Conclusion and Discussion}

At the basis of the results presented in this paper is the supercover of a continuous object. The supercover of a continuous object is formed by all the discrete points whose corresponding voxel "touches" the continuous object. The link with previous results of discrete geometry is made by the fact that the supercover of a continuous plane is a discrete analytical plane [5]. The discrete supercover polygon is defined as the supercover of a $3 \mathrm{D}$ polygon defined in the continuous world. 
By defining a 3D line segment as the supercover of a continuous line segment, we are able to define edges for the supercover polygon. These discrete edges have similar properties than the continuous ones : they are independent of the orientation of the polygon and so two different supercover polygons can share a common edge. Another important property of the supercover polygon is that it is tunnel free.

This 3D discrete polygon is an interesting alternative to the classical 3D polygon [4] for the digitization of a continuous polygon. Many topological properties of the continuous polygon are preserved. We think that the supercover polygon could be a good choice for a complex discrete scene modelization for applications like discrete flight simulation, discrete ray tracing, etc.

However, several important questions remain open. The first question concerns the arithmetical thickness of the Supercover polygon. In certain cases, the supercover polygon is one point thicker than the optimal thickness needed to obtain a tunnel free polygon. Is it possible to define a Supercover polygon that is always of optimal thickness? The definition of a corresponding 3D discrete line is the key issue here. We haven't found any satisfactory answer so far. Another open question is : Is it possible to find a polygon definition that is simply 2-dimensional separating ? It is not very difficult to define a 2-dimensional separating polygon as plane segment of a 2-dimensional tunnel separating plane (called naive plane by J-P. Réveillès), but we loose most of the properties of the Supercover polygon such as compatible edge definition, etc. These and other questions need to be solved before we will be able to build a discrete analytical modeling environment compatible with classical continuous analytical modeling environments.

\section{References}

1. E.ANDRES and C.SIBATA, Discrete hyperplanes in arbitrary dimensions, submitted to CVGIP-GMIP.

2. E.ANDRES and C.SIBATA, Choice of Integer Part Function in Computer Graphics, submitted to IEEE TVCG.

3. D.COHEN and A.KAUFMAN, Fundamentals of surface voxelization, CVGIPGMIP, vol. 57, n.6, Nov.95.

4. A.KAUFMAN, An algorithm for 3D scan conversion of polygons, Proc. of Eurographics'87, AMSTERDAM, 1987.

5. J-P.REVEILLES, Geometrie Discrete, calcul en nombres entiers et algorithmique, State Thesis, Universite Louis Pasteur, STRASBOURG, Dec. 1991. 